\title{
Review of: "Study on gene knockout mice and human mutant individual reveals absence of CEP78 causes photoreceptor and sperm flagella impairments"
}

\author{
Xiao-Jin $\mathrm{He}$
}

Potential competing interests: The author(s) declared that no potential competing interests exist.

The paper "Study on gene knockout mice and human mutant individual reveals absence of CEP78 causes photoreceptor and sperm flagella impairments" is an interesting study trying to focus on monogenic factor related to multisystem syndrome. The study presented by discovers Cep78 as a new causative gene of CRD and male infertility in human and mice, which extends the phenotype of this disease-causing gene. This may contribute to the understanding of reproductive pathologies in men with ciliopathies. Cep78 knockout mice phenocopies the CEP78 lost patient, clearly that the authors have found a new causative gene for this new type of multisystem disease, which is a very meaningful discovery. These findings will contribute to further management and genetic consulting of patients with this genetic disorder. Moreover, the authors found that Cep78 regulates retina connecting cilia assembly, centriole length and sperm flagella formation, and provides evidence that Cep78 functions via forming a trimer with two intraflagellar transport proteins Ift20 and Ttc21a. The data are of high quality with novel findings, the rationale of each experiment is clear, and the article and figures are well-organized. In general, the results support the authors' conclusions. I have the following suggestion to improve the manuscript:

1. Line 85-86. The authors should add references to the statement "However, occasionally, CRD can also occur as a syndrome in combination of abnormalities of sperm flagella".

2. In Fig $3 \mathrm{~N}$ and Supp Table S2, the authors presented the quantitative MS data. They have identified many differentially regulated proteins upon Cep78 depletion. However, they simply showed a GO analyses of the proteins. Since cep78 is a cilia and centrosome protein, the author may want to compare the protein list with cilia database- "Mick, David U et al. "Proteomics of Primary Cilia by Proximity Labeling." Developmental cell vol. 35,4 (2015): 497-512." and centrosome database- “Gupta, Gagan D et al. 'A Dynamic Protein Interaction Landscape of the Human Centrosome-Cilium Interface.'Cell vol. 163,6 (2015): 1484-99." to make the analysis more targeted.

3. Spermatogenesis is a complicated multi-cellular developmental process. The authors have studied the function of cep78 in spermatocytes and round spermatozoa, but the effect of Cep78 deletion upon sertoli-cells and spermatogonia remains unknow. It is suggested that the author test markers ( e.g. Sox9 for sertoli celsl and Lin28a for spermatogonia) to explain whether the deletion of cep78 affects the development of sertoli-cells and spermatogonia.

4. The language of the manuscript should be carefully reviewed and revised. Several errors are listed 
below:

Line 31. The word "And" should be removed.

Line 36. "Conducted to" should be "conducted on".

Line 42. "Protein abnormalities" should be "abnormally expressed proteins".

Line 82. "Different" should be "distinct".

Line 118: "cilliogenesis" should be "ciliogenesis".

Line 118: "regulated" should be "regulates".

Line 119: "leaded" should be "lead". 